\title{
The Understanding of the Derivative Concept in Higher Education
}

\author{
Claudio Fuentealba ${ }^{1 *}$, Edelmira Badillo ${ }^{2}$, Gloria Sánchez-Matamoros ${ }^{3}$, Andrea Cárcamo ${ }^{1}$ \\ ${ }^{1}$ Facultad de Ciencias de la Ingeniería, Universidad Austral de Chile, Valdivia, CHILE \\ 2 Departament de Didàctica de la Matemàtica i de les Ciències Experimentals, Universitat Autònoma de Barcelona, Barcelona, \\ SPAIN \\ ${ }^{3}$ Departamento de Didáctica de las Matemáticas, Universidad de Sevilla, Sevilla, SPAIN
}

Received 1 June 2018 - Revised 17 August 2018 - Accepted 27 September 2018

\begin{abstract}
The aim of this work was to identify and characterize the levels of development of derivative schema. In order to do so, a questionnaire to 103 university students with previous instruction in Differential Calculus was applied. The questionnaire was composed of three tasks. For the identification of the levels of development of schema and their subsequent characterization, we consider the framework proposed by the APOS theory. In particular, this framework was operationalized through the establishment of 27 variables that allowed for the breakdown of the resolution protocols from the questionnaire into discrete elements. In this way, we obtained a vector associated with each of these variables. The identification of students assigned to each level of development of schema was carried out by a cluster analysis. Subsequently, we performed a statistical analysis of frequencies and implicative, with the 27 variables, which allowed to characterize the levels of development identified.
\end{abstract}

Keywords: derivative schema, levels of development, cluster analysis, implicative analysis, calculus

\section{INTRODUCTION}

Calculus is one of the greatest and most important achievements of the human intellect, and is a hallmark of the development of mathematics today, whose power and flexibility may be seen in its ability to reduce complex problems to rules and simple procedures in the most diverse areas of knowledge, such as mathematics, physics, engineering, social sciences and biology, among others (Berry \& Nyman, 2003; Kleiner, 2001).

In this sense, Ferrini-Mundy and Lauten (1994, p.120) describe Calculus as: “Calculus is a critical landmark in the mathematical preparation of students intending to pursue nearly all areas of science" For his part, Tall (1997, p. 289) states that Calculus "is both a climax of school mathematics and a gateway to further theoretical developments", which makes it a transitional point between elementary mathematics and advanced mathematics.

Despite the relevance of Calculus, one problem that has still remained unresolved is how to achieve understanding by university students in the fundamentals concepts of this course. Moreover, Calculus is typically considered a difficult subject for university students; it is noted that these students often can solve problems involving the proper application of rules or algorithmic procedures, but nonetheless have difficulties when they have to solve non-routine problems involving the understanding of concepts (Selden, Selden, Hauk, \& Mason, 1999), or the application of that understanding to real-world problems (Tall, 1992).

In particular, this study focuses on the concept of derivatives, which is one of the central and structural elements of any Calculus course, and is also a fundamental tool in the study and understanding of phenomena that involve changing or varying magnitudes (Vrancken \& Engler, 2014). Therefore, it corresponds to a basic concept applicable to many other fields in university curricula in mathematics, engineering, and other sciences.

Despite the importance of the derivative concept, the results of research related to understanding they find that this is very complex, showing a significant amount of university students who only manages to achieve a partial

(C) 2019 by the authors; licensee Modestum Ltd., UK. This article is an open access article distributed under the terms and conditions of the Creative Commons Attribution License (http://creativecommons.org/licenses/by/4.0/).

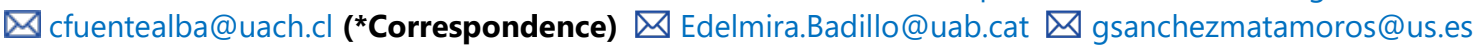




\section{Contribution of this paper to the literature}

- This research describes the quantitative analysis of data that are generally approached by qualitative methods.

- The results of this research confirm and extend conclusions obtained through qualitative studies on the development of the derivative schema.

- The methodological design proposed in this research can serve as a model for the analysis of schemas of different mathematical concepts.

understanding of this concept (Asiala et al., 1997; Baker, Cooley \& Trigueros, 2000; Cooley, Trigueros \& Baker, 2007; Ferrini-Mundy \& Graham, 1994; Fuentealba, Sánchez-Matamoros, Badillo \& Trigueros, 2017; Orton, 1983; SánchezMatamoros, 2004; Sánchez-Matamoros, Garcia \& Llinares, 2006, 2008; White \& Mitchelmore, 1996).

An aspect that has caused difficulties in the understanding of the derivative concept, relates to the use of teaching practices that have favored the learning of algorithmic methods by students (Artigue, 1995). This predilection for teaching algorithmic procedures makes students show serious difficulties and errors when faced with solving tasks that require understanding the meaning of the derivative, either through its analytical expression, as the limit of incremental ratio, or of its geometrical interpretation as slope of the tangent line (Baker et al., 2000; Cooley et al., 2007; Sánchez-Matamoros et al., 2006; Sánchez-Matamoros et al., 2008).

The problem of understanding of the derivative concept, though not new, is still one of the biggest challenges of mathematics education at the university level, and is a constant concern for institutions of higher education as it leads to low grades, high rates of failure, and the abandonment in Calculus courses (Bressoud, Mesa, \& Rasmussen, 2015; Ferrini-Mundy \& Graham, 1991). Considering this situation and the social demand concerning research in mathematics education, not only analyzing the problems of the teaching and learning of the discipline, but also contributing to the solution, our focus in this research is to determine the following: how is the understanding of the derivative concept is developed in university students with prior instruction in Differential Calculus? What are the characteristics of the different levels of development of derivative schema? Thus, we intend to deepen the understanding of how the concept of derivatives is formed in the minds of students in order for this information to contribute in the future to solving the problems associated with learning.

\section{BACKGROUND AND THEORETICAL FOUNDATIONS}

The understanding of concepts is an important goal for mathematics education, and it is generally accepted that this occurs when the pieces of knowledge that make up a concept are mentally connected together (Hiebert \& Carpenter, 1992). As students face new situations and experiences, they interact with your existing knowledge (von Glasersfeld, 1983), thus allowing such connections are made. New ideas can be integrated into existing schema or mindsets of the students, and can make the existing knowledge reconnect in new ways (Siegler, 1986). Understanding is seen in this way as a connected network, and pieces of knowledge that are built over time through the interaction of prior knowledge with new information.

In particular, this work has been considered a cognitive approach that, like the above ideas, consider the understanding of a mathematical concept as a gradual process of building and relationship between cognitive structures. As its epistemological reference, this approach takes constructivist ideas based on the genetic psychology of Piaget, which have been considered by Dubinsky and a group of researchers known as "Research on Undergraduate Mathematics Education" (RUMEC), who have developed a theoretical framework known as APOS theory (action-process-object-schema). This framework is the result of the interpretation of Piagetian ideas concerning reflective abstraction.

To understand how the APOS theory operates, it is important to note that the principle of reflective abstraction was considered by Piaget as the main mechanism for all mental construction, as well as the mechanism through which all logical-mathematical structures can develop in the mind of an individual (Arnon et al., 2014). According to Piaget, this principle has two parts:

The first part involves reflection, in the sense of awareness and contemplative thought, about what Piaget called content and operations that content, and in the sense of reflecting content and operations from a lower cognitive level or stage to a higher one [...]. The second part consists of the reconstruction and reorganization of the content and operations on this higher stage, that results in the operations themselves becoming content, to which new operations can be applied (Piaget, 1973; quoted in Arnon et al., 2014, p. 6)

In the APOS theory, actions, processes, objects and schema are mental structures that, according to this framework, an individual constructs when learning a particular mathematical concept, while the passage through these stages is not necessarily sequential (Trigueros, 2005). The mechanism to move from one state of construction of mathematical knowledge to another, in this theory, is reflective abstraction, which is a mental tool or device used in 


\section{Schema}

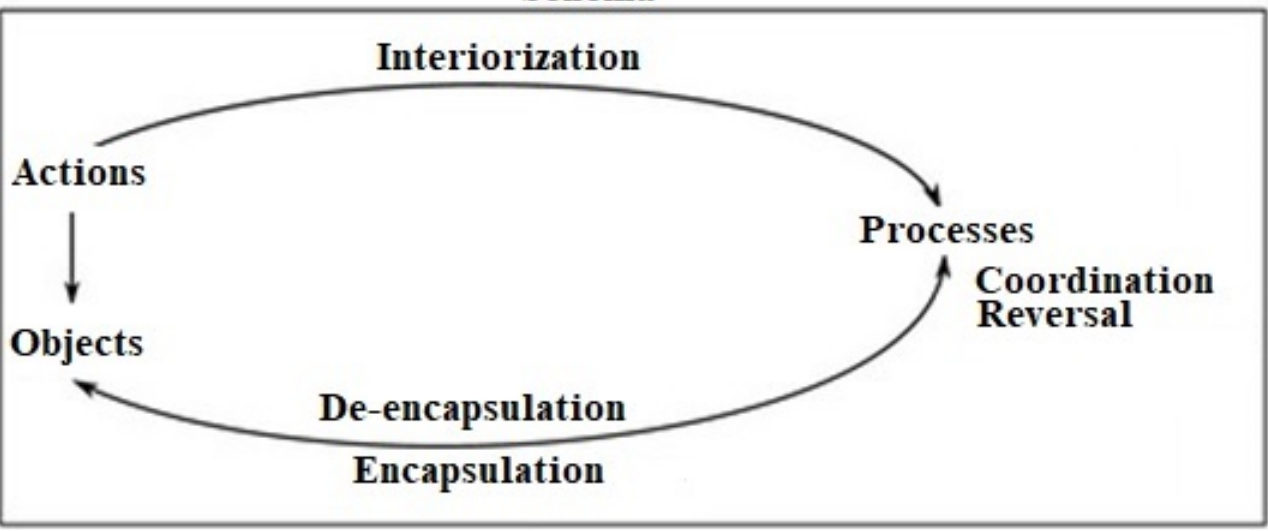

Figure 1. Structures and mental mechanisms involved in understanding a mathematical concept (based on Arnon et al., 2014, p. 18)

the process of knowledge construction, which allows the student to infer their properties on the basis of actions on objects, or to infer the relationships between objects of the same level of thinking. This implies, among other things, the organization of information in an intellectual framework organized higher-level (Dubinsky, 1991).

One of the hypotheses on which the APOS theory is based is that the construction of a new concept is based on the transformation of previous concepts, for which reason these concepts should initially be perceived by the individual as objects. Therefore, an action is a transformation of objects (previously constructed), perceived by the individual and external, in the sense that each step of the transformation requires explicit targeting and also needs an external stimulus to execute (Arnon et al., 2014). The structure of action is considered as the simplest within the APOE theory, but no less important, because it is essential in the construction of any mathematical concept. Also, a process is considered an internalized action, that is to say "mental"; in which the individual is aware and has control over the transformation produced by the action. This is characterized by the ability to imagine, skip, or reverse the steps involved in this transformation, without the need of an external stimulus. Interiorization is the mechanism that allows for the change of structure, from action to process, which is accomplished by repeating reflection on the actions (Arnon et al., 2014). A process can not only be generated by the interiorization of actions, but they can also be constructed from the coordination and reversal mechanisms of processes. When an individual is aware of the process and is able to conceive how it can be transformed as a whole by applying actions or processes, it is then said that the process has been encapsulated in a cognitive object (Arnon et al., 2014; Asiala et al., 1996). The mechanism associated with this change of state of the process is called encapsulation. Nevertheless, once a process is encapsulated in an object, if the individual is required to return to the process that gave rise to the object, this can be done by the de-encapsulation mechanism.

Finally, the last cognitive structure proposed by APOS theory is called a schema. A schema of a mathematical concept in particular is a coherent collection of actions, processes, objects and even other schemas and their interrelationships, grouped consciously or unconsciously in the mind of an individual, which can be used in the solution of a situation or mathematical problem involving the concept in question. The coherence of the schema is referred to as the individual's ability to recognize situations in which the schema is applicable and which are not (Trigueros, 2005).

The schema is regarded as a dynamic and complex cognitive structure that is constantly developing and evolving as the individual learns. Although sometimes it is generally thought that this structure is just beginning to form once the objects (due to progression of action, process, object and schema) are constructed, construction may begin even at the time the individual performs actions.

Although the mental structures (action, process, object and schema) of an individual during of an individual cannot be directly observed during the learning process, these structures can be inferred from the observation on what the individual can do, or not to face a particular situation or mathematical problem (Dubinsky, 1991). Thus, through observation and analysis, it is possible to characterize the stage of construction of a specific mathematical concept in which an individual may be found. Figure 1 shows the relationship between the mechanisms and the mental structures mentioned above.

Trigueros (2005) indicates that when a student is facing a specific problem in the field of mathematics, the student conjures up a schema to address the resolution. Upon evoking it, that student brings those built structures and relationships that he has at that time into play. Faced with the same task, different students can use different 
structures and different relationships between them. Thus, when considering the relationships established between the built structures, different levels of development of the schema can be identified in the responses of students who meet the same task or set of tasks. To address and characterize these differences in further developing the dynamism of the schema, APOS theory proposes the study of the development of a schema through the use of the triad of Intra, Inter and Trans, proposed by Piaget and Garcia (1983), which classifies it in one of these stages, depending on the level of relations that an individual can establish between the schema components and other cognitive structures. Following this idea, Piaget and Garcia (1983) define the levels of development of a schema as follows:

- Intra: this level is characterized by the discovery of any operative action, and the pursuit of analyzing its various internal properties or its immediate consequences, but with a two-part limitation. First, there is no coordination of this pre-operation with others in an organized grouping; but also, the internal analysis of the operation involved is accompanied by progressively corrected errors and gaps in the inference that can be derived from it (p. 163).

- Inter: once an initial step is comprised, it is possible to deduce the operations that are involved, or coordinate with other more or less similar ones for the creation of systems that involve certain transformations. While there is a new situation here, there are nevertheless limitations that arise from the fact that the compositions are restricted because they can only proceed with contiguous elements (p.165).

- Trans: This level is easily defined in terms of the above as involving, in addition to the transformations, a synthesis between them. This synthesis arrives at the construction of "structures" (p.167).

From this definition of the triad of the level of development of a schema, in recent decades, various studies have been made concerning the development of schemas. For example, research by Baker et al. (2000) analyzed the calculus graphing schema through the solutions given by students of a non-routine a graphic problem, in which it was not the expression of the function but rather a set of analytical conditions of that function that was presented. In their study, they posited that the Calculus graphic schema was formed by the interaction of two schemas that they called "property schema" and "interval schema". For his part, Badillo, Azcárate and Font (2011) uses an idea similar to that posed by Baker et al. (2000) for an analysis of the understanding of the concept of derivatives in a group of five mathematics and physics teachers from Colombia. The idea of coordination of schemas used in their research, considering that the derivative schema was formed by coordinating the "algebraic schema" and "graphic schema".

For the specific case of this study, reference was made to work by Sánchez-Matamoros (2004), in which an analysis of derivative schema in terms of logical relationships (conjunction, contrapositive, and equivalence) that students establish between different mathematical elements when solving problems. Therefore, consistent with the study by Sánchez-Matamoros (2004), it is understood that a mathematical element is "the product of the dissociation or segregation of the concept relating to the concept and its properties" (Piaget, 1973, p. 72). From this definition, it may be indicated that the derivative concept possesses structural elements of a different nature, characterized by the modes of representation and the character of these elements. With regard to the modes of representation, this paper considers that the concept of the derivative consists of two types of elements: analytical and graphic elements. Also, regarding their character or nature, it considers elements of a specific nature, if these elements refer to a specific property at a point, or apply overall, as appropriate, to a range corresponding to a property. Thus, it is understood that a schema corresponds to the mathematical structure formed by the mathematical elements and logical relationships established between them, and that can be evoked for solving a problem (Sánchez-Matamoros, 2004). From the identification of mathematical elements and logical relationships established between them, Sánchez-Matamoros (2004) defined, through a qualitative analysis:

- Intra-derivative level. No logical relationships are established between the mathematical elements (either graphical or analytical, specific or overarching), and the possible outlines of the relationship (the logical conjunction type) between them was made erroneously. Use of mathematical elements in isolation, and sometimes incorrectly.

- Inter-derivative level. Logical relationships are established between the mathematical elements used, but with limitations, the predominating using logical conjunction and relate only specific and/or overarching mathematical elements that are in the same mode of representation, analytical or graphic. More mathematical elements are used correctly than in the previous level.

- Trans-derivative level. Increases the repertoire of the use of the logical relationships (and logic, contrapositive, logical equivalence) between mathematical elements. At this level, the "synthesis" of modes of representation occurs. This leads to the construction of the mathematical structure. (p. 73-74)

Regarding the synthesis portion of the description of the level of development, this applies to situations in which it is necessary to relate (make logical connections) with graphic and analytical information, that is, using information from both imaging systems to consider together and arrive at a "thing" that was not known. "Considering the information together" means establishing some sort of logical relationship between mathematical 
elements to make a decision concerning the situation that currently exists (Sánchez-Matamoros, 2004). In this paper, the descriptors for each level of development based on mathematical elements and logical relationships for defining the study variables presented in the study design were considered.

Also, another important aspect in the development of schemas related to the issues raised by Piaget and Garcia (1983) on the gradual growth of the schema and the nature of the triad. In particular, these authors point out that:

The nature of the elements of the triad is functional rather than structural. Therefore, they follow a necessary order, since the development of Trans, as a system of transformations together in entirely new properties, involves the formation of some of these transformations at the Inter level, and that this latter group involves the knowledge of characters analyzed in the Intra (p. 171).

This last idea has been adapted by the APOS theory to analyze how a schema of a specific mathematical concept is developed, which for the case of this study corresponds to the derivative, in addition, allows to characterize the different levels of schema development, and how these relate to each other, which correspond to the goals of this research.

\section{METHODOLOGICAL DESIGN}

This work is part of a wider investigation; whose purpose is to analyze the derivative schema in college students with prior instruction in Differential Calculus. The methodological approach adopted is of mixed type with exploratory and confirmatory character, we worked with qualitative data and statistical analysis (Rocco, Bliss, Gallagher \& Perez-Prado, 2003). In this part of the work, we have focused on quantitative analysis.

\section{Participants}

The participants in this study were 103 college students from the academic years 2014/2015 and 2015/2016, with double majors in Mathematics and Physics at a public university in the province of Barcelona. All students had taken and passed at least one subject which included the topics of Differential Calculus. The option of choosing students who had already completed one or more courses in Differential Calculus is intentional and is based on two aspects: (1) our interest in characterizing the levels of development of the derivative schema, reached by college students after the instructional process; and, (2) the difficulty associated with the characterization of the encapsulation mechanism of processes, demonstrated in previous studies (Cooley et al., 2007; García et al., 2011; Font, Trigueros, Badillo \& Rubio, 2016; Fuentealba et al., 2017; Sánchez-Matamoros, 2004).

With regard to the characteristics of the participants, it may be mentioned that there is great variability in their age range, prior training, and academic level.

\section{Instrument}

The instrument used (see Figure 2) and discussed in this article is a questionnaire that was constructed by adapting three tasks used in previous research on the derivative concept (Baker et al., 2000; Cooley et al., 2007; Fuentealba, Sánchez-Matamoros \& Badillo, 2015; García et al., 2011; Sánchez-Matamoros, 2004; Sánchez-Matamoros et al., 2006). For it to be solved, it was necessary to use distinct mathematical elements that make up the derivative concept. This questionnaire was administered to 103 participants in this study and lasted approximately 90 minutes. 
Task 1

Description of aspects associated with its solution

Problem statement

Sketch the graph of a function $f$ satisfying the following conditions

a) $f$ is continuous on its domain

b) $f(2)=0$.

c) $f^{\prime}(2)=f^{\prime}(5)=0$

d) $\lim _{x \rightarrow-\infty} f(x)=-4$

e) $\lim _{x \rightarrow-8^{-}} f(x)=-\infty$

f) $f^{\prime}(x)<0$ when $5<x<8$

g) $f^{\prime}(x) \geq 0$ when $x<5$

h) $f^{\prime \prime}(x)<0$ when $3<x<8$

i) $f^{\prime \prime}(x)>0$ when $x<3$

The first task provides analytical information for the function $f$ in terms of $f, f^{\prime}$ and $f^{\prime \prime}$. With these conditions students are asked to sketch the graph of the function $f$. The objectives of this task are, on the one hand, to observe if students are able to establish both local and global relations between actions and processes in their schema, that associate the sign of $f^{\prime}$ in an interval with the process which allows to infer from the behaviour of the rate of change $f$ in that interval; the process associated with the sign of $f^{\prime \prime}$ in an interval with the process which allows the concavity of $f$ to be determined; and the process that associates the change of sign of $f^{\prime}$ with the possible existence of extreme values or inflection points. On the other hand, to observe if the student is able to identify the contradictions in the analytical conditions posed in the problem that would provide evidence of the construction of stronger relations between the elements that constitute the schema.

Task 2

\section{Description of aspects associated with its solution}

The second task, presented in graphical mode, has two parts, the first focuses on the local behaviour of the function and students are asked to calculate the derivative of $f$ at specific points. The objective of this part is to observe if the students are able to carry out the actions or processes required to calculate the derivative at the points where the function has a different behaviour. In the second part, they are requested to sketch the graph of $f^{\prime}$ knowing the graph of $f$. We wanted to determine if students had constructed the derivative function as an object, and if they were able to coordinate the processes involved in the management of all the information, local and global, in an analytical context, with those corresponding to the graphical context. Notice that in task 1 a logical implication is involved, while in this task the reciprocal implication needs to be used.

\section{Task 3}

\section{Description of aspects associated with its solution}

Problem statement

The following graph corresponds to the derivative of $f$. Sketch the possible graphs for $f$ and explain your reasoning

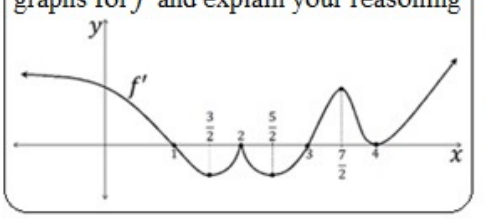

In the third task, students were given the graph of the function $f^{\prime}$, which includes several sign changes; increasing and decreasing slopes, zeros, extreme values and a corner point. The task asks to sketch a graph for the function $f$ and its objective is to assess whether students could coordinate the processes related to the extreme values of $f^{\prime}$ ' with the processes associated with the extreme values and inflection points in $f$. Similarly, to coordinate the processes associated with the sign of $f^{\prime}$ with those related to the monotony of $f$ and finally, to coordinate the processes related to the increase of $f^{\prime}$ with those related to the convexity of $f$.

Figure 2. Tasks proposed in the questionnaire and description of aspects associated with its solution

\section{Analysis Methods}

As an initial step before performing the analysis, the resolutions protocols were discretized. For the discretize of these protocols, and to obtain a vector associated with each of them, 27 variables were defined (see Figure 3). These variables are the result of the breakdown of mathematical elements in both modes of representation 


\begin{tabular}{|c|c|}
\hline $\begin{array}{c}\text { Mathematical } \\
\text { element }\end{array}$ & Variable \\
\hline $\begin{array}{l}\text { Derivative at a } \\
\text { point } f^{\prime}(a)\end{array}$ & $\begin{array}{l}\mathrm{V}_{1} \text {. Correctly uses the geometric meaning of the derivative in } x=a \\
\mathrm{~V}_{2} \text {. Correctly uses the analytic meaning of the derivative in } x=a\end{array}$ \\
\hline $\begin{array}{l}\text { Derivative } \\
\text { function } f^{\prime}(x)\end{array}$ & $\begin{array}{l}\mathrm{V}_{3} \text {. Correctly uses the derivative function meaning } \\
\mathrm{V}_{4} \text {. Correctly uses the derivative operator meaning }\end{array}$ \\
\hline $\begin{array}{l}\text { Extreme value } \\
\text { of } f\end{array}$ & $\begin{array}{l}\mathrm{V}_{5} \text {. Correctly uses the geometric meaning of local maximum } \\
\mathrm{V}_{6} \text {. Correctly uses the analytic meaning of local maximum } \\
\mathrm{V}_{7 .} \text { Correctly uses the geometric meaning of local minimum } \\
\mathrm{V}_{8 .} \text { Correctly uses the analytic meaning of local minimum }\end{array}$ \\
\hline $\begin{array}{l}\text { Inflection point } \\
\text { of } f\end{array}$ & $\begin{array}{l}\mathrm{V}_{9} \text {. Correctly uses the geometric meaning of inflection point } \\
\mathrm{V}_{10} \text {. Correctly uses the analytic meaning of inflection point }\end{array}$ \\
\hline $\begin{array}{l}\text { Logic } \\
\text { equivalence } \\
\text { relation between } \\
\text { the sign of } f^{\prime} \\
\text { over an interval } \\
I \text {, and the } \\
\text { montony of } f \text { in } \\
\text { that interval }\end{array}$ & $\begin{array}{l}\mathrm{V}_{11} \text {. Correctly uses the logic implication relation between: the positive sign } \\
\text { of } f^{\prime} \text { over an interval and the strict increase of } f \text { in that interval } \\
\mathrm{V}_{12} \text {. Correctly uses the logic implication relation between: the strict } \\
\text { increase of } f \text { over an interval and the positive sign of } f^{\prime} \text { in that interval } \\
\mathrm{V}_{13} \text {. Correctly uses the logic implication relation between: the negative sign } \\
\text { of } f^{\prime} \text { over an interval and the strict decrease of } f \text { in that interval } \\
\mathrm{V}_{14 .} \text { Correctly uses the logic implication relation between: the strict } \\
\text { decrease of } f \text { over an interval and the negative sign of } f^{\prime} \text { in that interval }\end{array}$ \\
\hline $\begin{array}{l}\text { Logic } \\
\text { equivalence } \\
\text { relation between } \\
\text { the sign of } f^{\prime \prime} \\
\text { over an interval } \\
I \text {, and the } \\
\text { curvature of } f \\
\text { in that interval }\end{array}$ & $\begin{array}{l}\mathrm{V}_{15} \text {. Correctly uses the logic implication relation between: the positive } \\
\text { sign of } f^{\prime \prime} \text { over an interval and the convexity of } f \text { in that interval } \\
\mathrm{V}_{16} \text {. Correctly uses the logic implication relation between: the convexity } \\
\text { of } f \text { over an interval and the positive sign of } f^{\prime} \text { in that interval } \\
\mathrm{V}_{17} \text {. Correctly uses the logic implication relation between: the negative } \\
\text { sign of } f \text { " over an interval and the concavity of } f \text { in that interval } \\
\mathrm{V}_{18 .} \text {. Correctly uses the logic implication relation between: the concavity } \\
\text { of } f \text { over an interval and the negative sign of } f \text { " in that interval }\end{array}$ \\
\hline $\begin{array}{l}\text { Non-derivability } \\
\text { points of } f\end{array}$ & $\begin{array}{l}\mathrm{V}_{19 .} \text {. Correctly uses of the lateral derivatives } \\
\mathrm{V}_{20} \text {. Correctly uses the meaning the conflictive points (cusp points and } \\
\text { corner points) }\end{array}$ \\
\hline $\begin{array}{l}\text { Continuity and } \\
\text { derivability of } f\end{array}$ & $\begin{array}{l}\mathrm{V}_{21} \text {. Correctly uses the direct relation: if } f \text { is differentiable in } x=\mathrm{a} \text {, then } \\
f \text { is continuous in } x=a \\
\mathrm{~V}_{22} \text {. Correctly uses the contrapositive relation: if } f \text { in non-continuous } \\
\text { in } x=a \text {, then } f \text { is non-differentiable in } x=a\end{array}$ \\
\hline $\begin{array}{l}\text { Other variables } \\
\text { that were } \\
\text { observed }\end{array}$ & $\begin{array}{l}\mathrm{V}_{23 .} \text {. Can divide correctly a graph in different intervals determined by the } \\
\text { graphic elements provided (monotony and curvature) } \\
\mathrm{V}_{24 .} \text { Can define correctly different intervals of the function domain } \\
\text { determined by the analytic elements provided (signs and zeros) } \\
\mathrm{V}_{25} \text {. Can graph correctly a function from knowledge of its graphic } \\
\text { properties } \\
\mathrm{V}_{26} \text {. Can graph correctly a function from knowledge of its analytic } \\
\text { properties } \\
\mathrm{V}_{27} \text {. Can establish correctly relations between } f^{\prime} \text { and } f^{\prime \prime}\end{array}$ \\
\hline
\end{tabular}

Figure 3. Variables used for the discretization of the resolution protocols of each of the questionnaires

(analytical and graphical), the use of logical relationships and previous studies using the APOS theory (Fuentealba et al., 2017; Fuentealba, Badillo \& Sánchez-Matamoros, in press; Trigueros \& Escandón, 2008). For example, the decomposition of the logic relationship of double implication between the positive sign of the first derivative in an interval and the strict growth of the function in said interval, allowed us to generate the variables $V_{11}$ and $V_{12}$. Also, by decomposing of some mathematical elements in both modes of representation, we generated other variables, for example, the variables $V_{9}$ and $V_{10}$, associated with correct use of the meaning of inflection point. 
Table 1. Hierarchical Cluster obtained by varying the distance and the type of grouping selected

\begin{tabular}{ccc}
\hline Distance & Cluster grouping type & Cophenetic correlation \\
\hline Squared Euclidean & Complete linkage & 0,859 \\
\hline Euclidean & Complete linkage & 0,747 \\
\hline Manhattan & Complete linkage & 0,660 \\
\hline Squared Euclidean & Single linkage & 0,723 \\
\hline Euclidean & Single linkage & 0,775 \\
\hline Manhattan & Single linkage & 0,723 \\
\hline
\end{tabular}

The purpose of establishing these variables was to conduct a cluster analysis that would identify and characterize the levels of development of the derivative schema (groups delivered by conglomerates). However, to quantify the presence or absence of each of the variables in the resolution protocols used by the students, it was necessary to use a measurement scale to assign a score to each of them. For the specific case of this study, a scale of a binary type, 1 or 0 ( 1 in the case that the correct use of variables was observed, and 0 in another case). From these two tools (variables and scale) we obtained for each resolution protocol a vector of the type $\left(V_{1}, V_{2}, V_{3}, \ldots V_{27}\right)$, wherein each variable has a value of 0 or 1 . Each of these vectors was labeled with the letter $E$ and a subscript that indicates the student belonging to it, thus 103 vectors $\left(E_{1}, E_{2}, E_{3}, . ., E_{103}\right)$ were obtained.

Later, after three sub-matrices of certain data (Intra, Inter and Trans) were obtained through a cluster analysis, a frequency analysis was conducted with percentage frequencies in terms of the proper or improper use of the variables. However, this type of analysis does not indicate what the most important variables are within each level of development, and also allows for the relationships to be viewed between them. Therefore, to observe the underlying structures of the group of variables, an implicative statistical analysis (Analyze Statistique Implicative, or ASI, in French) is carried out on each of the levels, corresponding to a method of analysis which allows, from a set of data which interrelates with a population of subjects or objects with a set of variables, for the extracting and structuring of knowledge in the form of rules and generalized rules (Zamora, Gregori \& Orús, 2009). In particular, an implicative statistical analysis is a method of data mining, which is not symmetrical, and which allows to statistically model the quasi-implication $a \Rightarrow b$, that is to say, it attempts to quantify how likely it is to happen if the variable $b$ has been observed in the population variable E (Lerman, Gras \& Rostam, 1981). According to Trigueros and Escandón (2008), in this methodology, "the implication $a \Rightarrow b$ shall be admissible if the number of individuals $E$ that contradict it is very small, in probabilistic terms, in relation to the number of individuals expected under the hypothesis of having no relationship. If this happens, you can say that $A$, the set of observations to satisfy the feature, is "almost" contained in $B$, the set of observations that satisfy the characteristic $b$ " (p. 67). Unlike data based symmetrical analysis methods, for example, on a distance or a correlation, the sets of rules sets obtained by the analysis can lead to implicative causal hypotheses (Zamora et al., 2009).

\section{ANALYSIS AND RESULTS}

As mentioned, the first step corresponded to the embodiment of the cluster analysis with 103 vectors obtained by the discretization of the questionnaires. It is important to note that cluster analysis does not present a single solution, but rather the result of this depends on the characteristics of the selected process, i.e., the distance used and agglomerative or clustering method. While this study is intended to characterize the levels of development of the derivative schema, it was assumed, indicating the APOS theory as to the levels of development of an outline of any mathematical concept are three and that under this framework are the triad Intra, Inter and Trans (Arnon et al., 2014; Piaget \& Garcia, 1983). Given the foregoing, it was built with the Infostat 2016 application six different conglomerates combining distances (Euclidean, squared Euclidean and Mahattan) and clustering methods (simple and complete linkage). Values of the cophenetic correlations for each of the six hierarchical clusters obtained are presented in Table $\mathbf{1}$.

Considering the results of the six conglomerates that were constructed and their cophenetic correlations we choose those whose cophenetic correlation coefficient was highest, which in this case corresponded to 0.859 , which was selected. The rationale for this decision was based on the issues raised by Sokal and Rohlf (1962), who indicate that this ratio ensures a correct rating when its value is close to one.

As a result of the cluster analysis with the squared Euclidean distance and the complete agglomerative linkage method, the dendrogram shown in Figure 4 was obtained. 


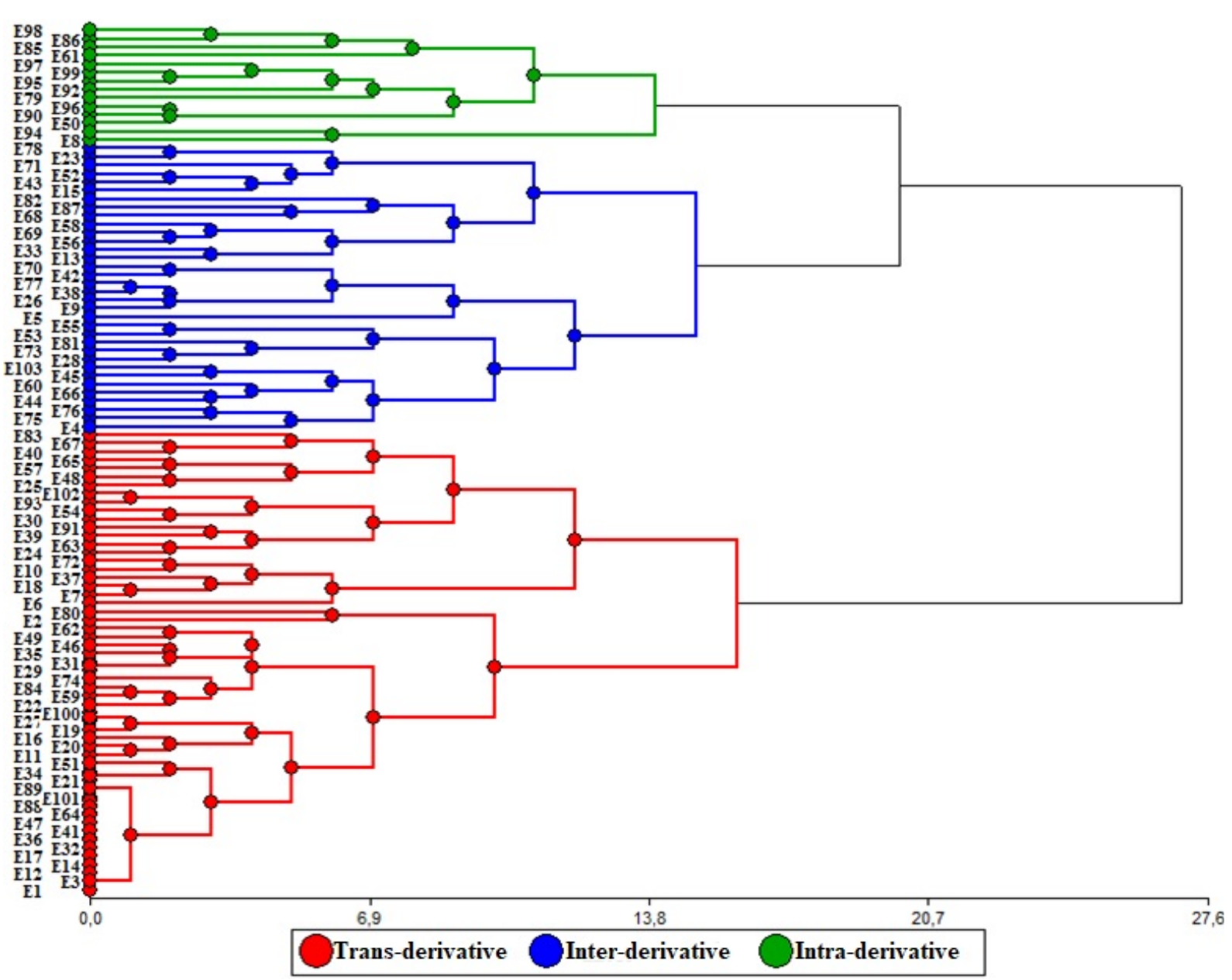

Figure 4. Complete dendrogram obtained with squared Euclidean distance and complete linkage

Table 2. Students assigned to the levels of development of the derivative schema

\begin{tabular}{|c|c|c|c|}
\hline Level & Trans-derivative & Inter-derivative & Intra-derivative \\
\hline Students & $\begin{array}{l}\text { E1, E2, E3, E6, E7, E10, E11, E12, } \\
\text { E14, E16, E17, E18, E19, E20, E21, } \\
\text { E22, E24, E25, E27, E29, E30, E31, } \\
\text { E32, E34, E35, E36, E37, E39, E40, } \\
\text { E41, E46, E47, E48, E49, E51, E54, } \\
\text { E57, E59, E62, E63, E64, E65, E67, } \\
\text { E72, E74, E80, E83, E84, E88, E89, } \\
\text { E91, E93, E100, E101, E102. }\end{array}$ & $\begin{array}{l}\text { E4, E5, E9, E13, E15, } \\
\text { E23, E26, E28, E33, E38, } \\
\text { E42, E43, E44, E45, E52, } \\
\text { E53, E55, E56, E58, E60, } \\
\text { E66, E68, E69, E70, E71, } \\
\text { E73, E75, E76, E77, E78, } \\
\text { E81, E82, E87, E103. }\end{array}$ & $\begin{array}{l}\text { E8, E50, E61, } \\
\text { E79, E85, E86, } \\
\text { E90, E92, E94 } \\
\text { E95, E96, E97, } \\
\text { E98, E99. }\end{array}$ \\
\hline Total & 55 & 34 & 14 \\
\hline
\end{tabular}

The dendrogram obtained through the cluster analysis allowed for the data matrix to be divided into three submatrices, one for each level of development of the derivative schema. In particular, in Figure 4, the red portion of the dendrogram corresponds to students assigned by the cluster analysis at the Trans-derivative level of development, the blue portion corresponds to students assigned to the Inter-derivative level, and the green portion are students assigned to the Intra-derivative level.

The students classified in each of the levels of development of the derivative schema are presented in Table 2.

The division of the matrix allowed for a descriptive statistical analysis of the percentages of the correct use of the 27 variables for each of the levels of development of the schema. The percentages of correct use of variables for each level of development are presented in Table 3. 
Table 3. Frequency of correct use of the variables by mathematical criterion defining element and each variable

\begin{tabular}{|c|c|c|c|c|}
\hline \multirow{2}{*}{ Elements } & \multirow{2}{*}{ Criterion } & \multicolumn{3}{|c|}{ Percentage } \\
\hline & & Trans & Inter & Intra \\
\hline \multirow{2}{*}{ 1. Derivative at a point $f^{\prime}(a)$} & V1: Geometric meaning & 87 & 68 & 50 \\
\hline & V2: Analytic meaning & 58 & 29 & 7 \\
\hline \multirow{2}{*}{ 2. Derivative function $f^{\prime}(x)$} & V3: Derivative as a function & 51 & 0 & 0 \\
\hline & V4: Derivative as an operator & 73 & 24 & 24 \\
\hline \multirow{4}{*}{ 3. Extreme value of $f$} & V5: Local maximum geometrically & 98 & 65 & 79 \\
\hline & V6: Local maximum analytically & 98 & 94 & 94 \\
\hline & V7: Local minimum geometrically & 98 & 56 & 50 \\
\hline & V8: Local minimum analytically & 98 & 59 & 29 \\
\hline \multirow{2}{*}{ 4. Inflection point of $f$} & V9: Inflection point geometrically & 96 & 41 & 21 \\
\hline & V10: Inflection point analytically & 98 & 94 & 7 \\
\hline \multirow{4}{*}{$\begin{array}{l}\text { 5. Logic equivalence relation } \\
\text { between: the sign of } f^{\prime} \text { over an } \\
\text { interval } l \text {, and the monotony } \\
\text { of } f \text { in that interval }\end{array}$} & V11: $f^{\prime}>0 \rightarrow f$ increase strict & 100 & 100 & 0 \\
\hline & V12: $f$ increase strict $\rightarrow f^{\prime}>0$ & 75 & 47 & 21 \\
\hline & V13: $f^{\prime}<0 \rightarrow f$ decrease strict & 100 & 100 & 0 \\
\hline & V14: $f$ decrease strict $\rightarrow f^{\prime}<0$ & 75 & 41 & 21 \\
\hline \multirow{4}{*}{$\begin{array}{l}\text { 6. Logic equivalence relation } \\
\text { between: the sign of } f^{\prime \prime} \text { over } \\
\text { an interval } I \text {, and the curvature } \\
\text { of } f \text { in that interval }\end{array}$} & V15: $f^{\prime \prime}>0 \rightarrow f$ convex & 100 & 100 & 14 \\
\hline & V16: $f$ convex $\rightarrow f^{\prime \prime}>0$ & 56 & 6 & 0 \\
\hline & V17: $f^{\prime \prime}<0 \rightarrow f$ concave & 100 & 100 & 7 \\
\hline & V18: $f$ concave $\rightarrow f^{\prime \prime}<0$ & 71 & 56 & 50 \\
\hline \multirow{2}{*}{$\begin{array}{l}\text { 7. Non- differentiability points } \\
\text { of } f\end{array}$} & V19: Lateral derivatives & 80 & 29 & 29 \\
\hline & V20: Conflictive points & 75 & 0 & 7 \\
\hline \multicolumn{2}{|c|}{ 8. Continuity and derivability of $\mathrm{V} 21$ : If $f$ is differentiable in $x=a \rightarrow f$ is continous in $x=a$} & 96 & 62 & 64 \\
\hline$f$ & V22: $f$ is non-continous in $x=a \rightarrow f$ is non-differentiable in $x=a$ & 93 & 65 & 50 \\
\hline \multirow{5}{*}{ Other variables } & V23: Determine intervals to sketch a function from graphical information & 100 & 24 & 36 \\
\hline & V24: Determine intervals to sketch a function from analytical information & 100 & 94 & 21 \\
\hline & V25: Sketch a function from its graphic properties & 100 & 15 & 21 \\
\hline & V26: Sketch a function from its analytic properties & 62 & 41 & 0 \\
\hline & V27: Relations between $f^{\prime}$ and $f^{\prime \prime}$ & 40 & 0 & 0 \\
\hline
\end{tabular}

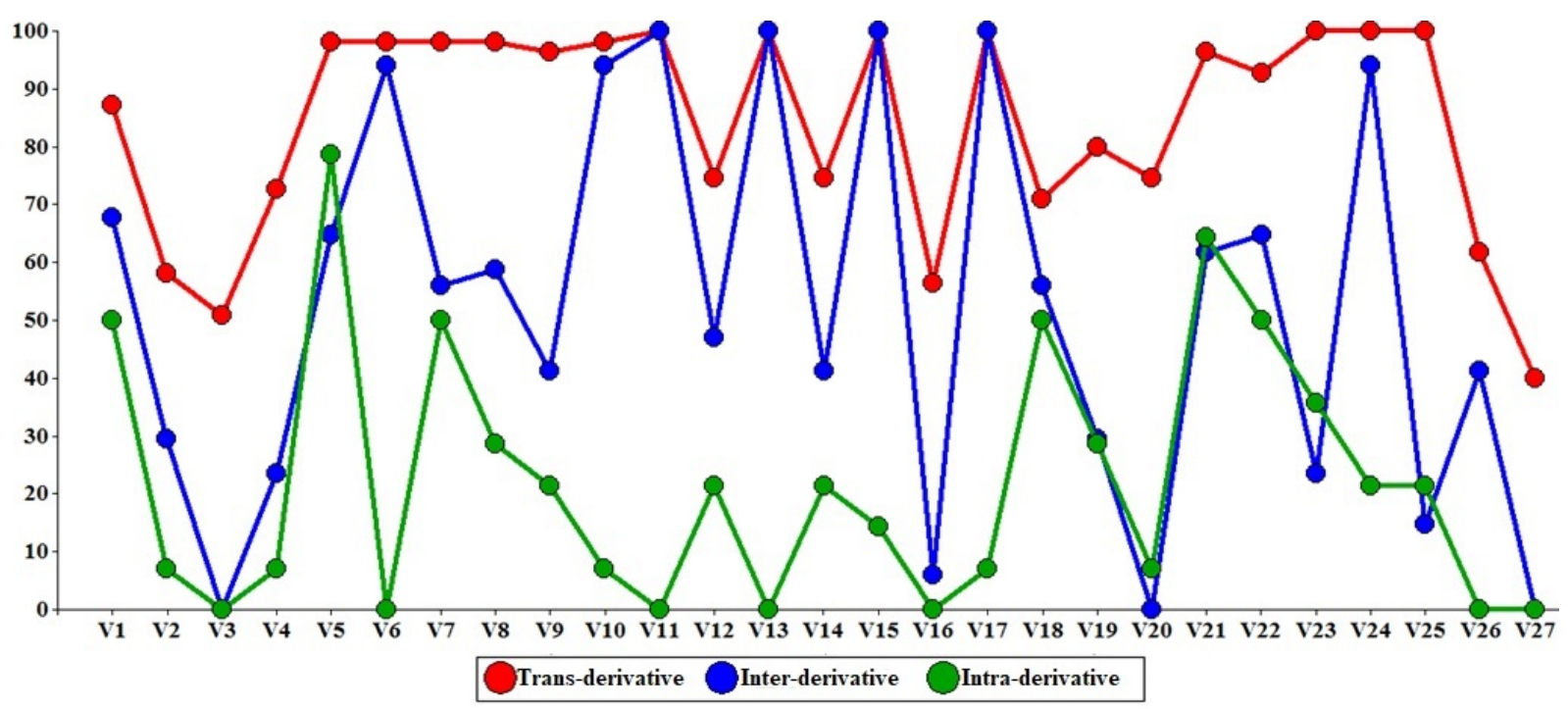

Figure 5. Graph of multivariant profiles levels development derivative schema

In order to more clearly visualize the differences and similarities between the three levels of development schema graph multivariate profiles are constructed from information percentages of correct use of the variables presented in Table 3. The multivariant graph profiles are presented in Figure 5.

Among the most important characteristics that can be inferred from Figure 5 are:

- Students at the levels of Intra-derivative (7\%) and Inter-derivative (29\%) have difficulties developing the interpretation of the derivative as the limit of the incremental ratio $\left(\mathrm{V}_{2}\right)$. Meanwhile, students at the Transderivative level use it correctly in $58 \%$ of cases. 
- Students at the Intra-derivative $(0 \%)$ and Inter-derivative $(0 \%)$ levels of development do not consider the derivative as a function $\left(\mathrm{V}_{3}\right)$. Conversely, more than $50 \%$ of students at the Trans-derivative level of development do.

- Students at the Intra-derivative $(7 \%)$ and Inter-derivative $(24 \%)$ levels have difficulty with the use of the derivative as linear operator $\left(\mathrm{V}_{4}\right)$. Meanwhile, $73 \%$ of students at the Trans-derivative level of development used this variable correctly.

- Students at the Trans-derivative level of development have no difficulty determining extreme values and inflection points $\left(\mathrm{V}_{5}, \mathrm{~V}_{6}, \mathrm{~V}_{7}, \mathrm{~V}_{8}, \mathrm{~V}_{9}, \mathrm{~V}_{10}\right)$, regardless of the representation mode in which the task is provided. Meanwhile, students at the Inter-derivative level do have some difficulties in determining extreme values and inflection points, especially when the information on the task is provided in graphing mode $\left(V_{5}, V_{7}, V_{9}\right)$. Similarly, students at the Intra-derivative level also present difficulties in determining extremes and inflection point values, but these difficulties are even greater than the level of Inter-derivative development, moreover, presented in both modes of representation.

- Students at the Trans-derivative level of development generally have no difficulty establishing direct and contrapositive relationships, between the sign of the first derivative and the monotony of the function $\left(\mathrm{V}_{11}\right.$, $\mathrm{V}_{12}, \mathrm{~V}_{13}, \mathrm{~V}_{14}$ ). Instead, students at the Inter-derivative level have difficulties in establishing contrary relationships, which is to say, between the monotony of the function and the sign of the first derivative $\left(V_{12}\right.$, $\mathrm{V}_{14}$ ). Meanwhile, students at the Intra-derivative level have difficulty in establishing both relationships (direct and contrapositive).

- Students at the Trans-derivative level have little difficulty in establishing direct and contrary relationships, between the sign of the second derivative and the curvature of the function $\left(\mathrm{V}_{15}, \mathrm{~V}_{16}, \mathrm{~V}_{17}, \mathrm{~V}_{18}\right)$. Instead, students at the Inter-derivative level have difficulty establishing contrary relationships, which is to say, between the curvature of the function and the sign of the second derivative $\left(\mathrm{V}_{16}, \mathrm{~V}_{18}\right)$. Meanwhile, students at the Intra-derivative level of development have serious difficulties in establishing these relationships (direct and contrapositive)

- Regarding the use of the lateral derivatives $\left(\mathrm{V}_{19}\right)$ and the processing of conflict points $\left(\mathrm{V}_{20}\right)$, it is noted that students at the Trans-derivative level generally do not have difficulties in their proper use. However, students of the Intra-derivative and Inter-derivative levels do have serious difficulties in using both variables.

- Students at the Trans-derivative level do not show great difficulties in the use of direct and contrapositive relations between differentiability and continuity $\left(\mathrm{V}_{21}, \mathrm{~V}_{22}\right)$. Instead, while students at the Intra-derivative and Inter-derivative levels use them, they still have some difficulties in doing.

- Students at the Trans-derivative level can determine ranges from graphic information $\left(\mathrm{V}_{23}\right)$ and correctly create a function from this information $\left(\mathrm{V}_{25}\right)$ without difficulty. On the other hand, students at the Intraderivative and Inter-derivative levels have difficulty doing so. Similarly, the students at the Trans-derivative level intervals can also determine from analytical information provided $\left(\mathrm{V}_{24}\right)$ and can correctly create a function from this information $\left(\mathrm{V}_{26}\right)$ without difficulty. Students at the Inter-derivative level can also determine the ranges from analytical information, however, they show great difficulties in outlining a function with such information.

- Only some of the students at the Trans-derivative level of development are able to establish relationships between the first and second derivative.

While all information provided by the percentages regarding the correct use of variables and multivariate profiles is valuable to characterize the levels of development of the derivative schema, this does not provide information regarding which variables are most important and determine the underlying structure of each level of development, and thus, diagrams were constructed to obtain such implicative information for each of the levels of development. In Figure 6 shows the trees associated with each level. 

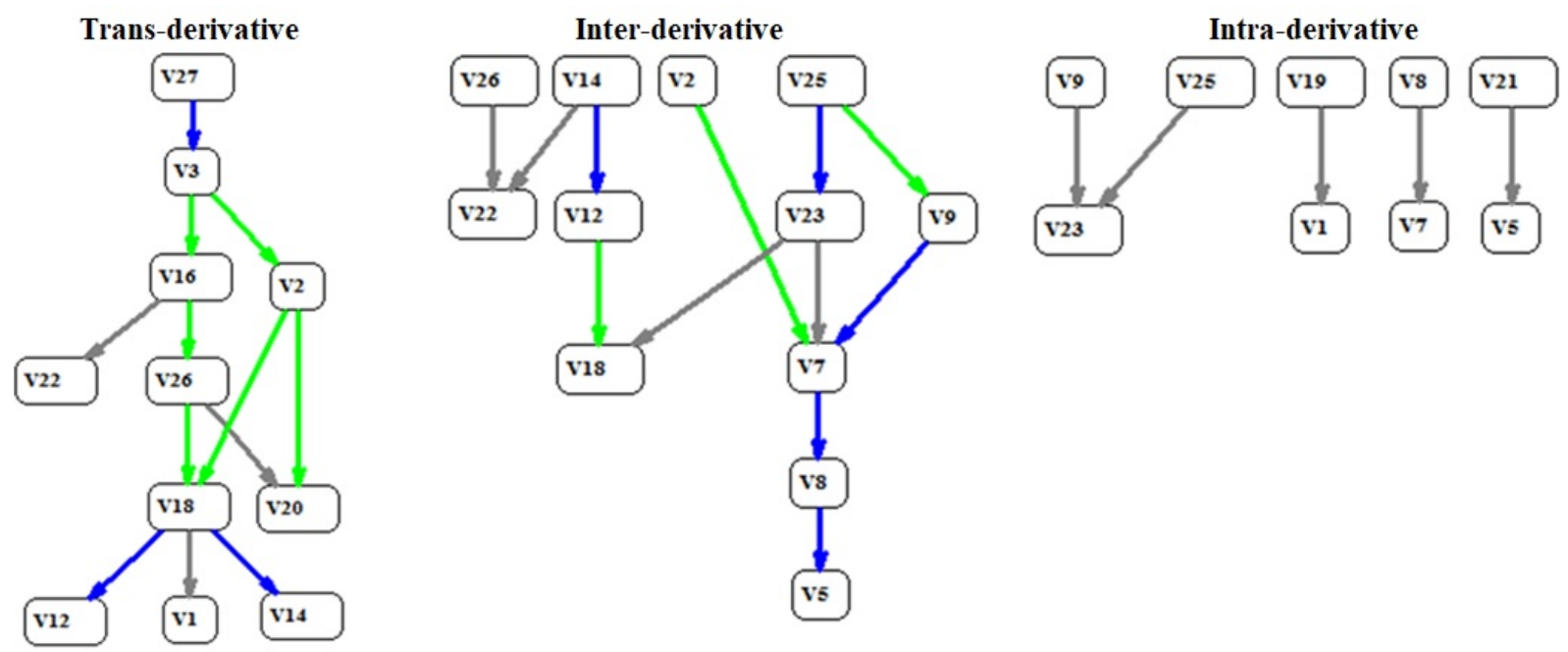

Figure 6. Implicative trees for each level of development of derivative schema

For each level of development of the schema, the corresponding implicative tree diagram provides information about the most important variables of the structure. In particular, the variables that begin implicative chains are those that cause more difficulties in each level, and the ending implicative variables correspond to chains that were easier to use. Therefore, if we consider the implicative tree for the Trans-derivative level of development, we can indicate that the most important variable is related to establishment of relations between the first and second derivative $\left(V_{27}\right)$; also this indicates that the students who are able to establish such relationships can also successfully use other variables. In addition, it is important to note that the different colors of the arrows indicate the strength of involvement. In particular, blue corresponds to implications with $95 \%$ significance, green with $90 \%$, and gray with $85 \%$. In addition, as we can see, not all variables are displayed on the implicative tree, which is due to the fact that the implications of these variables with the present the diagram are below $85 \%$ of significance.

Another important aspect of note is that the implicative tree of the Trans-derivative level, as already mentioned, only has an underlying variable that determines the structure of the relations between them. In addition, these relationships are significant, with a value that is $85 \%$ or higher. Meanwhile, at the Inter-derivative level of development, there are four variables that determine the structure of relations, and in addition, their implications values are greater than or equal to $85 \%$. In addition, differences may be noted in the number of levels of implications for each level of development. In particular, the Trans-derivative level has five levels of implication, the Interderivative has four levels of implication, and the Intra-derivative only has one level of implication. In addition, in this last level, no chain of implications is formed.

\section{DISCUSSION AND CONCLUSIONS}

The results of this work have allowed us to identify levels associated with the development of the derivative schema. Each level has the characteristics associated with the previous level and new ones that differentiate it, which shows the progressive nature of the understanding of the concept. This characterization of the different levels of development of the schema, conducted through a cluster analysis and the frequency and implicative analysis confirms several conclusions from previous qualitative studies done beforehand (Baker et al., 2000; Cooley et al., 2007; Sánchez-Matamoros, 2004; Sánchez-Matamoros et al., 2006), regarding the essential role they play; the modes of representation, the ends and inflection points values, as well as the logical relationships that may be established between mathematical elements, which are those that determine and differentiate the distinct levels of the development of the schema.

Moreover, the results of implicative analysis carried out in each of these levels of development of the schema also corroborate and extend the results of previous research. Thus, for example, in research Sánchez-Matamoros (2004), Sánchez-Matamoros et al. (2006) and Garcia et al. (2011) a student that is considered to be in the Trans level of development of the derivative schema is able to transfer all relations $f$ and $f^{\prime}$ to the pair $f^{\prime}$ and $f^{\prime \prime}$. This result is similar to that obtained in the implicative tree of the development level Trans-derivative of this work, which indicates that the most important variable is related to establishment of relations between the first and second derivatives $\left(V_{27}\right)$, this It shows that the student is able to establish these relationships can also successfully use the other variables $\left(\mathrm{V}_{1}, \mathrm{~V}_{2}, \ldots, \mathrm{V}_{26}\right)$. In addition, the implicative analysis using different levels of statistical significance (95\% blue, green $90 \%$, and $85 \%$ gray) and the quasi-implications provide certain information regarding the consistency of the construction of the schema. In particular, this coherence is observed in the number of variables 
that determine the implications of chains and the statistical significance of these, with the observation of a number of variables that underlie the structure of each level of development that increase from the Trans to the Intra level (one for the Trans, four for the Inter, and five for the Intra).

Also, the number of levels of involvement observed at each level of development also reinforces the conclusion that the coherence of the schema is only achieved at the Trans-derivative level of development, which in this case has five levels of implications, unlike what happens to the level of Inter-derivative development that has four levels of involvement, and the Intra-derivative level that only has one level of involvement.

Finally, this design and characterization of the levels of development of the derivative schema can be enhanced with a holistic view that incorporates the different meanings of the concept, such as those proposed by various studies that use the ontosemiotic approach (Font et al., 2016; Pino-Fan, Godino \& Font, 2011; Pino-Fan, Godino, \& Font, 2018). Likewise, we note that the methodological design used can be applied to identify and characterize levels and/or sublevels of the development of schemas of other mathematical concepts.

\section{ACKNOWLEDGEMENTS}

This work has been partially funded by Projects EDU2014-54526-R, EDU2015-65378-P, EDU2016-81994-REDT and EDU2017-87411-R (The Spanish Ministry of Economy and Competitiveness), by "SGR-2017-101" (GIPEAM, AGAUR) and the Facultad de Ciencias de la Ingeniería of the Universidad Austral de Chile.

\section{REFERENCES}

Arnon, I., Cottrill, J., Dubinsky, E., Oktaç, A., Fuentes, S. R., Trigueros, M., \& Weller, K. (2014). APOS theory: A framework for research and curriculum development in mathematics education. Berlin: Springer. https:/ / doi.org/10.1007/978-1-4614-7966-6

Asiala, M., Brown, A., DeVries, D. J., Dubinsky, E., Mathews, D., \& Thomas, K. (1996). A Framework for Research and Curriculum Development in Undergraduate Mathematics Education. Research in Collegiate Mathematics Education, 2, 1-32. https:/ / doi.org/10.1090/cbmath/006/01

Asiala, M., Cottrill, J., Dubinsky, E., \& Schwingendorf, K. (1997). The Development of Students' Graphical Understanding of the Derivate. Journal of Mathematics Behavior, 16(4), 399-430. https:/ / doi.org/10.1016/S0732-3123(97)90015-8

Badillo, E., Azcárate, C., \& Font, V. (2011). Análisis de los niveles de comprensión de los objetos f'(a) y f'(x) de profesores de matemáticas. Enseñanza de las Ciencias, 29(2), 191-206. https:// doi.org/10.5565/rev/ec/v29n2.546

Baker, B., Cooley, L., \& Trigueros, M. (2000). A calculus graphing schema. Journal for research in mathematics education, 31(5), 557-578. https:/ / doi.org/10.2307/749887

Berry, J., \& Nyman, M. A. (2003). Promoting students' graphical understanding of the calculus. Journal of Mathematical Behavior, 22(4), 479-495. https:/ / doi.org/10.1016/j.jmathb.2003.09.006

Bressoud, D.M., Mesa, V., \& Rasmussen, C. (2015). Insights and recommendations from the MAA National Study of College Calculus. New York: MAA Press.

Cooley, L., Trigueros, M., \& Baker, B. (2007). Schema thematization: A framework and a example. Journal for Research in Mathematics Education, 38(4), 370-392. https:/ / doi.org/10.2307/30034879

Dubinsky, E. (1991). Reflective abstraction in advanced mathematical thinking. In D. Tall (Ed.), Advanced mathematical thinking (pp. 95-126). Dordrecht: Kluwer.

Ferrini-Mundy, J., \& Graham, G. (1991). An Overview of the Calculus Curriculum Reform Effort: Issues for Learning, Teaching, and Curriculum Development. American Mathematical Monthly, 98(7), 627-635. https://doi.org/10.2307/2324931 https:// doi.org/10.1080/00029890.1991.11995769

Ferrini-Mundy, J., \& Graham, K. (1994). Research in calculus learning: understanding limits, derivates and integrals. In E. Dubinsky, \& J. Kaput (Eds.), Research Issues in Undergraduate Mathematics Learning, MAA Notes 33, (pp. 31-45). Washington DC: Mathematical Association of America.

Ferrini-Mundy, J., \& Lauten, D. (1994). Learning about calculus learning. The Mathematics Teacher, 87(2), 115-121.

Font, V., Trigueros, M., Badillo, E., \& Rubio, N. (2016). Mathematical objects through the lens of two different theoretical perspectives: APOS and OSA. Educational Studies in Mathematics,91(1), 107-122. https:/ / doi.org/10.1007/s10649-015-9639-6

Fuentealba, C., Badillo, E., \& Sánchez-Matamoros, G. (in press). Puntos de no-derivabilidad de una función y su importancia en la comprensión del concepto de derivada. Educação e Pesquisa. 
Fuentealba, C., Sánchez-Matamoros, G., Badillo, E. (2015). Análisis de tareas que pueden promover el desarrollo de la comprensión de la derivada. Uno: Revista de didáctica de las matematicas, (71), 72-78.

Fuentealba, C., Sánchez-Matamoros, G., Badillo, E., \& Trigueros, M. (2017). Thematization of derivative schema in university students: nuances in constructing relations between a function's successive derivatives. International Journal of Mathematical Education in Science and Technology, 48(3), 374-392. https:/ / doi.org/10.1080/0020739X.2016.1248508

García, M., Llinares, S., \& Sánchez-Matamoros, G. (2011). Characterizing thematized derivative schema by the underlying emergent structures. International journal of science and mathematics education, 9(5), 1023-1045. https:// doi.org/10.1007/s10763-010-9227-2

Hiebert, J., \& Carpenter, T. (1992). Learning and teaching with understanding. Handbook of research on mathematics teaching and learning: A project of the National Council of Teachers of Mathematics (pp. 65-97).

Kleiner, I. (2001). History of the infinitely small and the infinitely large in calculus. Educational Studies in Mathematics, 48(2), 134-174. https:/ / doi.org/10.1023/ A:1016090528065

Lerman, I., Gras, R., \& Rostam, H. (1981). Élaboration et évaluation d'un indice d'implication pour des données binaires. 2. Mathématiques et sciences humaines, 75, 5-47.

Orton, A. (1983). Students' understanding of differentiation. Educational Studies in Mathematics, 14(3), 235-250. https://doi.org/10.1007/BF00410540

Piaget J. (1973). Las estructuras matemáticas y las estructuras operatorias de la inteligencia. En La colección Psicología y Educación. La enseñanza de las matemáticas (pp. 3-28). Madrid: Editorial Aguilar.

Piaget, J., \& García, R. (1983). Psicogénesis e Historia de la Ciencia. México, España, Argentina, Colombia: Siglo Veintiuno Editores, S.A.

Pino-Fan, L., Godino, J., \& Font, V. (2011). Faceta epistémica del conocimiento didáctico-matemático sobre la derivada. Educação Matemática Pesquisa, 13(1), 141-178.

Pino-Fan, L., Godino, J., \& Font, V. (2018). Assessing key epistemic features of didactic-mathematical knowledge of prospective teachers: the case of the derivative. Journal of Mathematics Teacher Education, 21(1), 63-94. https:/ / doi.org/10.1007/s10857-016-9349-8

Sánchez-Matamoros, G. (2004). Análisis de la comprensión de los alumnos de bachillerato y primer curso de la universidad sobre la noción matemática de derivada. (Desarrollo del concepto) (Doctoral dissertation), Universidad de Sevilla, Spain.

Sánchez-Matamoros, G., García, M., \& Llinares, S. (2006). El desarrollo del esquema de derivada. Enseñanza de las Ciencias, 24(1), 85-98.

Sánchez-Matamoros, G., García, M., \& Llinares, S. (2008). La comprensión de la derivada como objeto de investigación en didáctica de la Matemática. RELIME, 11(2), 267-296.

Selden, A., Selden, J., Hauk, S., \& Mason, A. (1999). Do calculus students eventually learn to solve non-routine problems. Tennessee Technical University Department of Mathematics. Technical Report.

Siegler, R. (1986). Unities across domains in childrens strategy choices. In Minnesota Symposia on Child Psychology (pp. 1-48). Mahwah: Lawrence Erlbaum Assoc Inc.

Sokal, R. R., \& Rohlf, F. J. (1962). The comparison of dendrograms by objective methods. Taxon, 11(2), 33-40. https://doi.org/10.2307/1217208

Tall, D. (1992). The transition to Advanced Mathematical Thinking: Functions, Limits, Infinity, and Proof. Handbook of Research on Mathematics Teaching and Learning (pp. 495-514). New York: MacMillan Publishing Company.

Tall, D. (1997). Informatie technologie en Wiskunde Onderwijs, Niewe Wiskrant Functions and Calculus. In A. Bishop, M. Clements, C. Keitel, J. Kilpatrick, \& C. Laborde (Eds.), International Handbook of Mathematics Education (pp. 289-325). Dordrecht: Kluwer.

Trigueros, M. (2005). La noción de esquema en la investigación enmatemática educativa a nivel superior. Educación Matemática, 17(1), 5-31.

Trigueros, M., \& Escandon, C. (2008). Los conceptos relevantes en el aprendizaje de la graficación. Revista Mexicana de Investigación Educativa, 13(36), 59-85.

Von Glasersfeld, E. (1983). On the concept of interpretation. Poetics, 12(2-3), 207-218. https:/ / doi.org/10.1016/0304$422 \times(83) 90028-1$

Vrancken, S., \& Engler, A. (2014). Una Introducción a la Derivada desde la Variación y el Cambio: resultados de una investigación con estudiantes de primer año de la universidad. Bolema, 28(48), 449-468. https://doi.org/10.1590/1980-4415v28n48a22 
White, P., \& Mitchelmore, M. (1996). Conceptual knowledge in introductory calculus. Journal for Research in Mathematics Education, 27(1), 79-95. https:/ / doi.org/10.2307/749199

Zamora, L., Gregori, P., \& Orús, P. (2009). Conceptos fundamentales del Análisis Estadístico Implicativo (ASI) y su soporte computacional CHIC. Contribuciones al ASI, 4, 65-101.

\section{http://www.ejmste.com}

